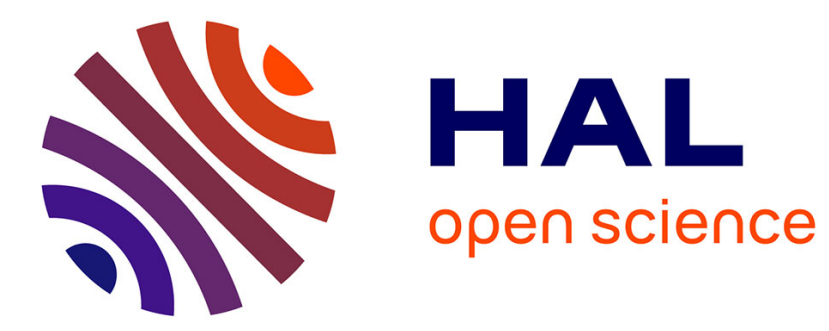

\title{
Lavage et patouillage: de la baignoire dans l'album pour enfants
}

Cécile Boulaire

\section{To cite this version:}

Cécile Boulaire. Lavage et patouillage: de la baignoire dans l'album pour enfants. L'esprit créateur, 2005, French Children's Literature, XLV (4), pp.73-86. 10.1353/esp.2010.0229 . hal-01170176

\section{HAL Id: hal-01170176 \\ https://hal.science/hal-01170176}

Submitted on 1 Jul 2015

HAL is a multi-disciplinary open access archive for the deposit and dissemination of scientific research documents, whether they are published or not. The documents may come from teaching and research institutions in France or abroad, or from public or private research centers.
L'archive ouverte pluridisciplinaire HAL, est destinée au dépôt et à la diffusion de documents scientifiques de niveau recherche, publiés ou non, émanant des établissements d'enseignement et de recherche français ou étrangers, des laboratoires publics ou privés.

\section{(ㄷ)(1) $\Theta$}

Distributed under a Creative Commons Attribution - NoDerivatives| 4.0 International 


\section{Lavage et patouillage : De la baignoire dans l'album pour enfants}

Le bain semble tellement faire partie du quotidien du jeune enfant que personne ne s'étonne de voir représenter baignoires et scènes de bain dans les albums qui lui sont destinés. Pourtant derrière l'évidence se joue une partie complexe où motifs anthropologiques et symboliques interfêrent avec le discours éducatif hygiéniste. La puissance évocatrice de l'eau, l'objet même de la baignoire, récent si l'on s'en réfère à l'histoire des pratiques corporelles occidentales, la nécessaire nudité du baigneur ainsi que l'éventuelle mixité du moment de bain, voilà quelques-uns des enjeux qui font basculer lộlbum pour enfants du référentiel au littéraire.

Commençons par balayer quelques certitudes: le bain n'a pas pour [seule] vocation de se laver. Par ailleurs se laver ne passe pas forcément par le bain. Lôuvrage d'Anne Varichon, Le corps des peuples ${ }^{1}$, rappelle ainsi que les Indiens Papago du sud de l'Arizona nettoient leur corps en le frottant avec de la terre sableuse et que les tribus indiennes d'Amérique du nord utilisent un tipi de sudation. Par ailleurs les peuples pratiquant par tradition l'immersion du corps le font rarement pour de simples motifs hygiéniques, les ablutions s'inscrivant dans un rituel de purification qui engage l'être

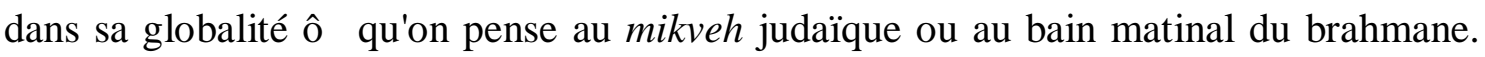
Gaston Bachelard note dans L'eau et les rêves que ñl'imagination matérielle trouve dans l'eau la matière pure par excellence, la matière naturellement pure ${ }^{2}$ ò. Enfin l'injonction de propreté corporelle dans notre société occidentale n'a pris que très tardivement la forme du bain. Georges Vigarello rappelle que c'est la méfiance à l'égard de l'eau pénètrant les corps, bien plus que des difficultés matérielles d'acheminement de l'eau, qui freine le développement des bains dans les usages domestiques ${ }^{3}$. De même le bain quotidien du nourrisson est d'invention tardive, et les manuels de puériculture qui circulent dans la France du premier XXe siècle le décrivent avec une rigueur et des précautions qui en font un rituel quasi mystique ${ }^{4}$. Ainsi rien ne va de soi dans ce qui est pourtant devenu un temps fort de la journée du jeune enfant. Une pratique apparemment simple et sans enjeu que les albums pour enfants, depuis le début des années 1930, vont évoquer parfois dans le seul souci de refléter le quotidien, mais bien souvent aussi en la réinvestissant de toute sa force symbolique. 
A ma connaissance, l'une des premières baignoires de livres d'enfants apparaît dans l'Histoire de Babar le petit éléphant que Jean de Brunhoff publie au Jardin des Modes en $1931^{5}$. On se souvient que l'éléphant, rendu orphelin par le meurtre de sa mère, fuit vers la ville, où il va peu à peu se "civiliser" avant de revenir dans la grande forêt se faire couronner roi. La processus de socialisation de Babar est triple: il s'agit de quitter la sauvagerie animale pour s'humaniser, de quitter l'enfance pour l'âge adulte, enfin d'intégrer les manières d'être de la bourgeoisie. En quelques pages Babar adopte la station debout, se détourne de la vitrine des jouets et du jubilatoire ascenseur pour s'acheter un costume "d'une agréable couleur verte". Il peut ainsi être accueilli chez "son amie la vieille dame", dont la fréquentation lui permettra de parachever son éducation. Sur la même double page Jean de Brunhoff distribue quatre images itératives qui nous présentent la nouvelle vie domestique de Babar: il sait se tenir à table, dormir dans un lit. Le matin, il accompagne la vieille dame dans une séance de gymnastique, puis il prend un bain. Jean de Brunhoff n'abuse pas des effets faciles que lui permettrait la corpulence de son personnage. Certes, la baignoire déborde quelque peu, mais le résultat en est moins comique que symbolique.

En effet, ce bain civilisé fait écho à la première double-page de l'album où des petits éléphants s'adonnent à tous les jeux de l'enfance. Parmi eux, huit se baignent dans une mare, s'arrosent, font la planche, en toute jouissance des corps en liberté. Page 19 au contraire, Babar en est réduit à user sagement de sa trompe, pressant une éponge; son corps de pachyderme est bien à l'étroit dans la baignoire des hommes. Renonçant à son animalité, il a perdu cette liberté des corps.

Dans le même temps il est entré dans la logique purement humaine qui oppose la nudité au vêtement. Babar ne peut être ñnuò qu'à partir du moment où il a une fois endossé un habit. Jean de Brunhoff ressent donc la nécessité de revêtir son personnage d'un slip rouge pour la séance de gymnastique; et pour le bain, Babar est véritablement nu $\partial$ peut-être notre premier héros nu ? Avant lui, on ne se lave pas à la vue des lecteurs. Max et Moritz ne se baignent pas après leur aventure dans la suie puis la farine ${ }^{6}$. Pire encore, le transgressif Struwwelpeter du docteur Heinrich Hoffmann refuse obstinément de se laisser couper ongles et cheveux ${ }^{7}$ ! Et si Boucle d'or énumère les divers ustensiles domestiques des trois ours, point de baignoire chez eux. Seule Elsa Beskow, en 1929, déshabille ses petits héros Pierre et Charlotte qui nônt pas résisté à lâppel du frais ruisseau : mais elle est suédoise, et ses albums ne seront connus en France que dans les années $1960^{8}$. L'Histoire de Babar témoigne donc d'une évolution 
des pratiques corporelles en même temps que l'album inaugure un nouvel espace symbolique dans l'ordre des représentations de l'univers enfantin ${ }^{9}$. Quelques pages plus loin, négligemment accoudé à la cheminée, Babar peut désormais animer les soirées bourgeoises de sa vieille amie. Dans ce parcours, l'immersion dans les eaux du bain marque profondément l'abandon de la sauvagerie animale pour l'entrée dans une humanité bourgeoise et hygiéniste.

Dans les mêmes années, les Albums du Père Castor, qui viennent de naître, offrent une autre image de bains. En 1934, Bonjour bonsoir propose aux enfants une série de cartons imagés par Nathalie Parain, sur un scénario de Paul Faucher. Il s'agit de présenter au jeune enfant des scènes quotidiennes, auxquelles il associera les ustensiles appropriés. Une image splendide de géométrisme montre le tout petit dans sa baignoire: une simple bassine de tôle émaillée, mouchetée de gris, comme on en trouve dans les familles modestes à cette époque. Un broc de tôle bleue est posé à portée de main. L'enfant, les yeux fermés en une attitude recueillie, se savonne consciencieusement le ventre au gant. Ce bain-ci témoigne avec clarté du projet éducatif du Père Castor. Reflétant les usages les plus communs (par opposition à la baignoire bourgeoise de la vieille dame), l'image présente au petit lecteur un reflet à la fois familier et positif. L'enfant de papier s'assume en se lavant sans aide; pas de mère pour le savonner. Paul Faucher s'ingéniait à encourager l'autonomie des enfants, qu'il souhaitait faciliter par ses livres. Son petit héros, tout à sa toilette, est bien sage ð trop sage? Le plaisir sensuel de l'immersion dans l'eau tiède, celui que redoutait encore le XIXe siècle ${ }^{10}$, ne semble pas l'atteindre. Nulle pensée voluptueuse sous la frange sombre de ce bébé; nul désir de patouilleé

C'est avec bien davantage de malice qu'André François fera prendre un bain à son crocodile en 1956. Les Larmes de crocodile, publié par l'audacieux Robert Delpire, est un clin d'î il amusé à Babar. Comme le grand album de Jean de Brunhoff, celui-ci adopte le format inhabituel de l'animal, une longue bande à l'italienne ${ }^{11}$. On prétend y expliquer à un enfant ce que sont les ñarmes de crocodileò. Prétexte en fait à évoquer une étrange chasse au crocodile du Nil, qu'on civilise pour en faire un délicat orateur de soirées mondaines. L'image pastiche celle où Babar, en frac, raconte ses aventures aux amis de son hôtesse. Ici le crocodile cabotine, coupe à la main, et l'incongruité de sa queue reptilienne ne semble pas rompre la fascination qu'il exerce sur les femmes en robe du soir. Mais cette soirée n'a été rendue possible que par la socialisation de la bête, qui passe là encore par la baignoire. Mal proportionné lui aussi, le crocodile n'est 
cependant pas aussi coincé que l'était l'éléphant. Il laisse tout bonnement dépasser sa queue de la baignoire et, en bon animal aquatique, semble jouir pleinement du moment, les yeux clos, tandis que le texte précise qu'il ñador[e] les bains d'eau tièdeò. Et tandis que Babar nostalgique accoudé à la fenêtre versait des pleurs sur sa mère et son enfance, notre crocodile s'ébat sans regrets dans son nouvel univers. S'il verse quelques larmes, ce ne sont que des larmesé de crocodile! Le savoureux ouvrage d'André François préfigure ce que sera l'album pour enfants dans la décennie suivante, quand le public, lassé des histoire policées et optimistes du Père Castor, découvrira avec un peu de stupeur les audaces nouvelles d'un Maurice Sendak ou d'un Tomi Ungerer $\succsim$ ce dernier pastichant lui aussi Babar à travers deux histoires dôdoption de bestioles inhabituelles, un serpent (Crictor) et surtout une pieuvre (Emile) à qui lôn donne un bain ${ }^{12}$ é

Car Paul Faucher, qui s'était donné pour mission de faire découvrir aux enfants ñe merveilleux de la natureò, ne se départit jamais de son positivisme. En 1960, à travers Perlette Goutte d'eau et son degoût de la pollution urbaine, il réaffirme sa préférence pour lậau courante et naturelle. Le magnifique Panier qui venait de la mer quôl signe en 1962 sur des images de Gerda Muller laisse éclater la force et la plénitude des corps d'enfants plongés dans les eaux revigorantes de la mer. Et sôl nôy a pas un seul bain dans les albums de la série Enfants de la terre, la petite tzigane Sarah ${ }^{13}$ est encouragée par son père à se baigner dans la rivière car, dit-il, ñcette eau, c'est de l'eau du Bon Dieu. Demain nous n'aurons peut-être que de l'eau des ñgadgéò, de l'eau prisonnière dans des tuyaux, celle-là n'est pas bonne.ò Une eau fraîche et courante, en pleine nature, pour la purification des corps vigoureux des enfants, voilà ce à quoi invitent les albums du Père Castor encore en pleine décennie 1970.

Progressivement des figures dôbord animales, puis anthropomorphes vont laisser voir un autre usage enfantin de la baignoire. Apparu en 1952 dans le magazine humoristique Punch, Max, le hamster malicieux inventé par P.L.Giovannetti, invite à voir dans la baignoire un instrument de l'épanouissement sans entraves du corps ${ }^{14}$. Dans l'une de ces péripéties visuelles, Max ñfait la baleineò. Puis deux bras agrippés aux rebords laissent les pattes libres pour un vigoureux pédalage qui asperge le sol. Enfin l'animal se hisse hors du bain, et finit d'inonder la pièce. Un autre ñstripò est plus explicite encore. Max hilare, assis dans son bain, s'arrose à l'éponge, puis fait mousser le savon avant de s'arrêter, visiblement en quête d'une idée plus à la (dé)mesure de la scène de bains. La dernière image le montre ravi, laissant jaillir des chapelets de bulles d'uneé cornemuse écossaise. A travers le hamster apparaît donc l'idée féconde d'un 
possible détournement des lieux de socialisation enfantine. Le bain ici lave peu, mais il est l'occasion d'une dépense d'activité infantile, improductive, jubilatoire.

Dans les mêmes années, une enfant cette fois, l'étourdissante Eloïse imaginée par K.Thompson et H.Knight s'invente une abominable tempête en pleine baignoire (aucun survivant), et nous ñsignale en passant qu'avec un gobelet en carton et une pomme de douche, on peut appeler la planète Mars ${ }^{15}$ ò. Quant à Tomi Ungerer, très sage encore dans cette fin des années 1950, il parvient pourtant à suggérer, dans Les Mellops trouvent $d u$ pétrole, une distinction capitale entre bain et douche. Certes, les deux instruments servent au lavage des corps, ici souillés par le forage d'un puits de pétrole. Mais tandis qu'en fond de pièce le papa cochon se douche sous le jet, la mère astique les trois cochonnets enfouis dans la mousse du bain (qui déborde). On pourrait penser que l'image ne fait que traduire le niveau d'équipement des ménages américains, douche et bain dans la même pièce. Plus profondément (mais encore sous couvert dônimaux), elle révèle le nouveau statut de la baignoire dans l'album pour enfants, espace d'investissement de la liberté individuelle infantile, régressive, voluptueuse et provocatrice.

Arnold Lobel est peut-être le premier à prendre le contre-pied de Jean de Brunhoff, avec les aventures de Porculus qui paraissent en $1968^{16}$. Le petit cochon est tout simplement victime de l'obsession de la propreté de sa maîtresse, fermière désoeuvrée au point de récurer jusqu'à la porcherie, cochon compris. Le savonnage de Porculus au baquet est représenté comme un viol de l'intimité du porcelet, négation même de son mode de vie: car Porculus aime par dessus tout s'enfoncer ñdans la bonne boue bien douceò. Après cette agression, il ne lui reste qu'à fuir. Plaidoyer en faveur du droit à l'individualité, Porculus inverse les valeurs avec drôlerie. Le cochon obtient d'être respecté dans ses préférences, au premier rang desquelles la saleté. Le voluptueux bain de boue répond à l'agression par l'eau et le savon. L'enfant n'est plus un simple sauvage à socialiser par le propre, il est un être autonome revendiquant son droit à la singularité. Et celle-ci ne s'affirme jamais aussi bien qu'à travers des plaisirs singuliers.

A partir de cette fin des années 1960, l'imaginaire de la baignoire et du bain se structure fermement autour de quatre valeurs: le plaisir des corps nus (qui laisse évidemment affleurer la sexualité); la nécessité d'une intimité garantie à chacun, enfants comme adultes; le désir de régression, parfois lié à la crainte primitive d'être dévoré; enfin le fantasme d'un débordement immaîtrisable des énergies enfantines. 
Certes, Babar comme le crocodile d'André François avaient sans doute plaisir à se plonger dans l'eau tiède, mais leur jouissance n'était pas la fonction première du bain qu'on nous offrait à voir. C'est étrangement en passant par la figure d'adultes qu'on met ce plaisir en avant. Les provocatrices éditions Harlin Quist vont ainsi successivement nous donner à voir une mère ${ }^{17}$ puis un lord anglais ${ }^{18}$ dans leur bain. Tous deux, bien que pudiquement dissimulés par le rebord de la baignoire ou le niveau de l'eau, sont bien évidemment nus dans leur bain. Or si Lord Zutalord peut apparaître comme un simple jouisseur, de surcroît doté de beaucoup de chance, puisque lors d'un tremblement de terre ébranlant son hôtel il descend sans encombres 14 étages sans quitter ni sa baignoire ni sa bonne humeur, le bain de la mère de Josette, dans le Conte $n^{\circ} 3$ de Ionesco, est plus franchement du côté de l'intimité amoureuse. Josette, comme chaque matin, s'immisce dans la chambre des parents: pénombre, draps froissés; le père est encore au lit, tandis que par la porte entrebaîllée de la salle de bains on aperçoit la mère dans son bain. C'est de toute évidence le secret de la chambre conjugale que vient troubler ici Josette, exigeant de rejoindre son papa au lit pour une histoire $ठ$ interrompue lorsque la mère en peignoir brise le tête-à-tête: "Allons, descendez du lit et habillez-vous".

Cet affleurement de la sensualité amoureuse à travers le bain de personnages adultes se retrouve lorsqu'en 1980 les éditions Des Femmes publient La grippe de Nils $^{19}$. Modernité oblige, l'album présente, par la voix d'une fillette, le quotidien de deux enfants vivant seuls avec leur mère, après la séparation des parents. Dès les premières pages la condition de la femme active est exprimée à travers cette injonction de la mère, de retour du travail: "Ecoutez les enfants. Pas un mot avant que je prenne un bain. Quelle purge ce métro. Je suis cre-vée!" Suit une voluptueuse image de bain où la mère, à demi immergée dans l'eau, laisse apparaître une généreuse poitrine, tandis la fillette et son jeune frère se déshabillent et la rejoignent pour une partie d'éclaboussures. Le texte de cette page fait très explicitement le lien entre cette nudité et la sexualité. L'enfant admire d'abord la poitrine de sa mère, qui peut ñfaire deux îlesò avec ses seins, puis elle évoque la curiosité d'une de ses camarades, contrainte à observer par le trou de la serrure si elle veut surprendre la nudité de sa propre mère, enfermée à clé dans la salle de bains; enfin la fillette en vient à évoquer Paul-Marie, délaissé par père et mère, et qui a profité de cette liberté pour raccompagner une camarade eté l'embrasser sur la bouche. Ici la nudité des enfants, fille et garçon, comme de la mère, est vécue avec 
naturel, mais le texte souligne dans le même temps que cette nudité au bain n'est jamais très éloignée des pensées voluptueuses de la sexualité.

Avec cette entrée des adultes dans l'univers jusqu'alors infantile du bain, permise par ces éditeurs avant-gardistes, la symbolique de la baignoire se complexifie. Le bain ñpour se laverò tolérait la présence d'un tiers, généralement la mère savonnante. Lorsque derrière le plaisir au bain affleure la volupté, la baignoire devient lieu d'intimité. Parallèle à la modernisation des équipements familiaux, une évolution caractéristique des années 1980 fait de la baignoire d'albums d'enfants un espace de réclusion, de faceà-face avec soi, de possible égoïsme. Ainsi en 1985 Felicia Bond nous raconte-t-elle l'histoire de Poinsettia ${ }^{20}$, petite cochonne accablée par une trop nombreuse fratrie, qui désire ardemment être seule. La coupe est pleine le soir où tous les lieux de possible intimité sont accaparés par l'un ou l'autre des petits cochons, notamment la baignoire dans la salle de bains rose bonboné Poinsettia fera en sorte d'être oubliée dans la maison déserte; elle pourra ainsi jouir, jusqu'à la nausée, de l'intimité de la baignoire. Sur une trame rigoureusement inverse, Théodore souris fils unique ${ }^{21}$ évoque les souffrances d'un souriceau trop solitaire, réjoui de l'arrivée du cousin Charlyé plaisir qui s'éteint rapidement lorsque Théodore se voit privé de ses plaisirs réservés, dont le bain rempli d'eau bien chaude. L'un et l'autre album, tout en favorisant le statu quo puisque chacun des héros finit par désirer ce qui lui était insupportable, institue le bain comme propice au retour de soi sur soi, dans un plaisir qu'on refuse momentanément de partager, et qui permet de s'affirmer en tant qu'individu.

De la même façon, l'énigmatique Moi, Matthieu, j'habite chez mon père ${ }^{22}$ publié par Ipomée en 1991, évoque avec finesse les questionnements d'un enfant tiraillé entre deux univers entre lesquels il peine à construire son identité. Pourtant l'épisode du bain, magnifiquement dessiné par Alain Gauthier, permet une pause dans cette lancinante quête de soi: ñChez Maman = chez moi, mon grand bol rouge m'attend / A la cuisine, c'est chocolat et phosphatine. / J'aime la salle de bains très chaude, / la mousse haute, la bergamote. / On se cherche dans la glace pleine de buée, / maman et moi Matthieu. / Du bout du doigt, je nous dessine tous les deux, là, / Et je signe: Matthieu, avec deux Tò. On lira la même nécessité de se retrouver avec soi, incarnée cette fois avec beaucoup d'humour par un personnage de mère, dans Le Bain de Madame Trompette ${ }^{23}$. La maman éléphante espère échapper à ses trois turbulents enfants en s'offrant un petit déjeuner tranquille dans la salle de bains: (r̃Elle met son bonnet de bain et se laisse glisser dans l'eau fumante, avec un soupir de plaisir. Puis elle verse du thé dans une tasse qu'elle 
pose en équilibre sur son ventre. Et elle ferme les yeux avec délices. C'est le paradisò). Hélas, le répit sera de courte duréeé Etre seul pour être soi, voilà ce que permet fugacement la baignoire, dans un quotidien par ailleurs mouvementé.

Pourtant la merveille de cette baignoire de livres consiste à être tout aussi bien lieu d'intimité et de recherche de solitude qu'espace de jeu collectif, temps de partage à nu. On sait combien la généralisation des bains, recommandés par les pédiatres, a modifié les règles sévères de séparation des sexes dans les familles. Pendant longtemps, chauffer suffisamment d'eau pour remplir une baignoire nécessite tant d'efforts qu'on fait baigner plusieurs enfants dans la même eau. La baignoire devient un lieu de proximité, des corps puis des êtres. D'où le bain de la mère et ses enfants dans $L a$ Grippe de Nils, ou la famille éclatée déjà cité, qui vient montrer la solidarité de cette famille pourtant dite ñéclatéeò: la baignoire semble ici une extension du ñgironò maternel. D'où encore ce bain qui clôt l'un des épisodes des Nouvelles Histoires d'Olivier Cochon $^{24}$ : la mère, fatiguée, réclame de rester seule un moment, à la grande incompréhension des cochonnets. Elle passe ainsi l'après-midi réfugiée dans un arbre du jardin tandis que les petits jouent dans la terre. La journée se termine par la proposition maternelle d'un bain ñtous les troisò, moment joyeux et délassant de réconciliation. Câst aussi le parti défendu avec un savoureux mauvais goût par Pef dans Rendez-moi mes poux en 1984: trop seul, abandonné à lui-même dans un appartement désert, Mathieu ne trouve de réconfort que dans la compagnie deé ses poux, quâl fait proliférer et pour lesquels il transformera la baignoire en piscine avec plongeoirs.

Partagé ou pris seul, le bain est avant tout lieu dồn plaisir sur la nature duquel il faut maintenant sônterroger. Si Poinsettia lit au bain, la jouissance prise par les autres baigneurs semble plaisir purement sensuel, parfois même légèrement régressif. Alors que depuis l'après-guerre le modèle de baignoire le plus répandu dans les logements modernes est encastré dans un caisson carrelé, nos baignoires de livres d'enfants empruntent dans leur grande majorité la forme archaïque de la cuve arrondie en fonte, posée directement sur pieds. Certes, l'iconographie de l'album a une forte tendance à l'archaïsme: combien de poêles à bois encore dans les cuisines de petites souris?é Ainsi la forme préférée, arrondie, enveloppante, de la baignoire ñà l'ancienneò tend à l'évocation graphique du ventre maternel $\partial$ le bain autorisant ainsi une régression vers l'aventure intra-utérine, entendue comme purement jouissive. Par ailleurs nos baignoires archaïques sont posées sur des ñpattesò évoquant explicitement l'animalité. Le bain devient ainsi plongée dans le ventre presque animal des premiers temps, régression vers 
les origines de la vie, tous êtres vivants confondus dans un même état primitif, aquatique: souvenons-nous du Têtard mystérieux ${ }^{25}$ de Steven Kellogg, dont l'une des étapes de la monstrueuse croissance sera la baignoire familiale, l'un des ñventresò successifs par lesquels passe cet embryon de saurien. Pas de canard dans ma baignoire $!^{26}$ se clôt aussi sur une double surprise soulignant la dimension plus mythique que référentielle de cette baignoire. Après plusieurs refus, la mère du héros a finalement accepté quôl rapporte du parc quelques î ufs de poissons. Au retour des vacances, les î ufs hébergés dans la baignoire sont devenus des dizaines de grenouilles, pour le plus grand bonheur de l'enfant, tandis que sấpouvante la maman dont on remarque enfin quâlle est elle-mêmeé enceinte.

La baignoire-ventre animal paraît donc un refuge protecteur. Dans le même temps, la séduction propre au bain laisse affleurer une terreur tout aussi ancestrale que l'est le fantasme de retour au liquide utérin: la dévoration. Ainsi $K i k i^{27}$ de Grégoire Solotareff raconte-t-il l'aventure aigre-douce dône souris trop solitaire ravie de l'arrivée de sa congénère Didi qui va s'occuper d'elle. Le premier acte de cette prise en main miattentionnée, mi-sadique est un bain un peu brusque: ñA la maison, Didi donne un bain à Kiki dans une tasse à déjeuner, pour s'amuserò. L'image témoigne du désarroi de Kiki, tandis que le texte laisse hésiter sur les causes de l'amusement: est-ce le fait de donner le bain, comme à une poupée? Ou plutôt l'incongruité du récipient choisi pour ces ablutions, explicitement destiné à la consommation alimentaire? L'inquiétude de Kiki semble tenir à l'impression de devenir consommable, ainsi infusée dans une tasse énormeé L'épisode n'est pas sans rappeler la fameuse Soupe à la souris inventée par Arnold Lobel en 1977, où une souris est capturée par une belette qui prétend la manger en soupe. La bestiole ne devra son salut qu'à la ruse. Claude Ponti a bien compris la secrète parenté de la marmite et de la baignoire, quand dans Pétronille et ses 120 petits $^{28}$ il fait kidnapper Pétronille par le sot Cafouillon qui la prend pour une souris verte. La voilà dès lors trempée, comme il se doit, dans l'huile puis dans l'eau. Or les récipients que dessine Ponti ressemblent curieusement à des baignoires, munis qu'ils sont de pattesé qui permettront l'évasion de l'impétueuse souris! Méfiance donc devant la baignoire: qui sait si le processus, en soi déjà désagréable, du récurage, ne dissimulerait pas une issue plus tragique encore?

De cette méfiance sourde naît un mouvement protéiforme de rébellion de l'enfant. Nombreux sont les personnages qui commencent par refuser d'aller au bain: ainsi Zaza, dans Zaza au bain ${ }^{29}$, Fabienne dans Un loup dans la baignoire ${ }^{30}$ ou encore 
le Max de Rosemary Wells ${ }^{31}$. Une fois dans le bain, le mouvement de refus symbolisant l'opposition à la discipline maternelle se transforme en énergie transgressive: puisqu'on ne peut échapper au bain, faisons tout au moins éclater ses règles. L'éclaboussure est la norme; pas un bain qui n'inonde le sol. Image jubilatoire d'Elzbieta dans Cornefolle ${ }^{32}$ où la vache rouge fait déborder une baignoire manifestement bien trop petite pour elle! Or Elzbieta écrit à propos de cet album ñ̃n optant pour la transgression, j'y suis allée hardiment, choisissant, pour sujet de mes attaques, ma propre mère.[é ] Il s'agissait donc, de manière métaphorique, de mettre en scène ma propre situation vis-à-vis de ma mère qui s'évertuait effectivement, telle Miss Patati aux prises avec Cornefolle, à me contenir de toutes les manières possibles et tout à fait abusivement puisque, constamment sur le qui-vive, j'étais on ne peut plus sage et, du moins extérieurement, totalement disciplinéeò ${ }^{33}$. Faire déborder la baignoire témoigne donc du rejet de la contention éducative.

Les albums les plus plats se contentent d'entériner complaisamment cette propension de l'enfant à éclabousser. Les artistes, eux, explorent la profondeur du motif. Le premier et l'un des plus inspirés est John Burningham, dans le magnifique Veux-tu sortir du bain, Marcelle? ${ }^{34}$ Burningham alterne pages de gauche au trait, rehaussée de ternes crayonnés, où la mère ressasse recommandations et reproches (ñJ'espère que tu n'as pas, une fois de plus, laissé le savon dans l'eau, n'est ce pas?ò), et pages de droite au graphisme intense, aux techniques riches et variées, dans lesquelles Marcelle échappe au réel par une fuite dans les canalisations et chevauche en un pays de rêve, où l'attendent mille aventures $ठ$ elle y défiera père et mère en de réjouissantes joutes nautiques. Cette évasion de l'enfant est triomphe métaphorique de l'imagination: s'il ne peut couper au bain, du moins le petit baigneur le transforme-t-il en puisant dans la volupté de l'eau chaude l'énergie d'une vigoureuse échappée de l'esprit.

Le bain est alors l'occasion de réaffirmer la dimension mystérieuse et fantasque de l'enfance, son irréductibilité aux codes et aux normes, son goût de l'esquive et de l'invention. Anaïs Vaugelade fait prendre un bain à son énergique Zuza ${ }^{35}$. Après deux double-pages où la fillette énumère les parties du corps qu'elle savonne surgit du baquet un appendice vert et recourbé que le texte cette fois ne nomme pas: ñZuza dans la baignoire se savonneé ò Hésitation sur l'appartenance de cette queue à l'anatomie de Zuza? L'album joue de cette inquiétude à la Carroll sur l'intégrité et l'animalité du corps de l'enfant. Rapide, muet, le petit album bascule de l'angoisse à la rivalité, puis à l'affrontement. Zuza sortira énergiquement le crocodile de son bain, avant de prendre 
finalement en pitié le reptile frissonnant, de nouveau admis au bain sous condition qu'il se rende utile. A la dernière page, Zuza réjouie se laisse savonner le bras par un crocodile à l'î illade coquine. Le bain mélange, transgresse, le bain laisse émerger la peur de l'étrange, le bain autorise la victoire du petit enfant sur la bête sauvage redevenue docile et presque sensuelle. Toujours triomphe la volupté. Dans le désopilant Une semaine de Monsieur Monsieur ${ }^{36}$ de Claude Ponti, autre album de petit format pour petites mains, ñSavonidi, Monsieur Monsieur prend un bain de pieds avec le crocodile de Zuzaò. La baignoire aux courbes langoureuses héberge ici crocodile et souris aux mêmes paupières alourdies par le plaisir, enfouis dans une mousse où surnagenté des pieds. Désir, plaisir et absurde mêlés.

Si l'on a vu abondamment de quoi était fait ce plaisir au bain, deux albums nous éclairent sur le désir qui sous-tend cette échappée aquatique. La fantasque et lumineuse Promenade au bord de l'eau ${ }^{37}$ d'Anne Brouillard débute au bord d'une baignoire pleine d'une eau miroitante. Un canard-jouet, comme échappé d'un album de John Burningham, flotte en avant de l'enfant, vers la tourne de la pageé C'est le prélude à un voyage onirique de canaux en rivières, à travers plaines et sous-bois, vers la mer du Nord. Nul n'a de clé pour cet album, errance vague au gré des souvenirs, des luminosités et des tentations. Et, à la différence de ce qui se passait pour Marcelle, l'album ne se clôt pas sur un retour au bain.

C'est un voyage à la fois plus ambitieux et moins débridé qu'entament Alice et Emile en vacances chez leur grand-père dans Laura perd la tête ${ }^{38}$. Ayant commencé leur bain par les amusements d'usage, les enfants voient leur bain prendre un tour singulier quand leur terre-neuve Laura saute dans la baignoire. Le basculement nous est signalé par une soudaine vue plongeante sur les trois personnages qui, voyant que l'eau déborde, quittent toute idée de maîtrise du phénomène pour choisir l'option inverse: ñFais-donc couler un peu plus d'eau!ò La salle de bain, puis l'appartement, puis l'immeuble entier seront inondés, les enfants quittant la grande baignoire encastrée immobile pour embarquer dans la baignoire de bébé. Surgissant de l'immeuble, le torrent rejoint la Seine, en direction de la mer $ð$ tandis qu'Alice s'inquiète soudain de sa nuditéé Isabelle Nières-Chevrel a bien dégagé la quête des origine qui fonde cette ñemontée aux sourcesò à la fois désirable et angoissante ${ }^{39}$. Il y a plus qu'une gigantesque patouille dans cette aventure, sans doute la force d'un désir qui rend nécessaire la transgression de toutes les limites. Et c'est le bain qui a suscité cette libération du fantasme. 
J'aimerais clore ce parcours sur un album qui ne contient pas de baignoire. Christian Bruel et Anne Bozellec publient en 1983 On serait des grenouilles aux éditions du Sourire qui mord, dans la collection ñPlaisirsò. Dans une cité un peu étriquée, aux jolis balcons années 1930, les enfants s'ennuient à la fenêtre: il pleut. Une double page glisse du rêve désirant à sa réalisation. Page de gauche, deux fillettes regardent tomber la pluie, pendant que la grenouille s'approche du rebord de son aquarium. Page de droite, la pluie a redoublé de force, les enfants ont disparu. La fenêtre entrouverte, corde à sauter abandonnée à la crémone, laisse s'échapper la grenouille. Le reste de l'album sera consacré à l'investissement, par les enfants, de la cité inondée transformée en inespérée piscine à ciel ouvert. On sort les bouées, les seaux, les canots. Des baquets s'improvisent navires, tandis que les enfants déshabillés prennent possession de l'arbre aux branches noueuses et s'abandonnent voluptueusement au plaisir de la baignade $\partial$ la vraie. Dans les dernières images quelques adultes, timidement, les rejoignent. La page de garde finale voit filer un voilier.

L'aventure est celle d'une jubilation sans entraves, plénitude du désir enfantin, transgression des règles comme du plausible, plaisir d'être corps: singe dans les branches, poisson dans l'eau. Jouissance parfaite, celle de cette fillette qui fait la planche, les yeux clos, tandis que la grenouille sourit de la même béatitude allongée sur une feuille, et l'abeille de même une branche plus haut. L'imaginaire de la baignoire est sublimé par cet album fluide tout au plaisir de l'invention débridée et de la convention atomisée. Une fillette rame encore, à l'aide d'un balai-brosse, dans son baquet-baignoire. Mais bientôt, comme les autres, elle abandonnera salopette et fichu et s'élancera elle aussi des branches de l'arbre vers l'eau claire. L'album de Bruel et Bozellec a déjà vingt ans. Il n'est pas sûr qu'on soit allé aussi loin depuis dans l'expression de la liberté créatrice de l'enfance. Pas sûr qu'on ait osé faire une telle confiance aux désirs sensualistes et exorbitants d'une enfance qui ne se contiendrait plus dans les limites trop lisses d'une baignoire émailléeé

Traverser cinquante années dôlbum pour enfants en France à bord dône baignoire aurait pu n⿳̣̂̂tre quâıne douce plaisanterie, si lôbjet ne sấtait révélé bien plus quânn ustensile quotidien voué au soin hygiénique de lậnfant. Neuf encore pour bien des foyers dans les années 1930, lôbjet sôffre presque vierge à lônvestissement de lâmaginaire. Or le livre pour enfants fait grand usage de symboles, et la baignoire, ronde et pleine de liquide chaud comme un ventre maternel, ventrue et pourvue de 
pattes comme un gros animal, galbée comme un navire, semble le véhicule idéal à des dérives que les pédiatres du début du siècle nânvisageaient sans doute pas. Adoptée par lôalbum pour enfants, elle révèle a posteriori lấvolution du statut de lânfant dans notre société. Alors que dans les années 1930 lôlbum accompagne le discours positif de socialisation du jeune enfant $\succsim$ intégration des codes bourgeois chez Babar, autonomie et action au Père Castor ð les années 1960 à 1980 voient progressivement émerger les revendications dôntimité et de plaisir, dans une atmosphère de rejet de la contention éducative. Moins en prise sur le réel, les années 1990 intensifient la dérision et paraissent adopter la démesure imaginative de lânfant, au risque parfois de lâffaiblir en la pastichant. Si la baignoire semble être finalement le lieu idéal où observer ce glissement dans la perception de lânfance, câst sans nul doute parce que lôbjet se situe à la jonction du référentiel pur (tous les enfants se lavent) et de lômaginaire littéraire. Son étude approfondie devrait idéalement sônscrire au carrefour de deux enquêtes encore à mener : une analyse du réalisme dans lâlbum; et une interrogation sur la place et le rôle de lấau dans les î uvres proposées aux jeunes enfants.

Cécile Boulaire

Université françois-Rabelais, Tours

Paru dans L'esprit créateur ; French Children's Literature, The John Hopkins University Press, Winter 2005, vol. XLV, n 4 (p.73-86).

\footnotetext{
${ }^{1}$ Anne Varichon, Le corps des peuples, us et coutumes de la propreté et de la séduction, (Paris: Seuil, 2003).

${ }^{2}$ Gaston Bachelard, L'eau et les rêves, Essai sur l'imagination de la matière p.181 (Paris: Corti, 1942).

${ }^{3}$ Georges Vigarello, Le propre et le sale, l'hygiène du corps depuis le Moyen Age (Paris: Seuil, 1985).

${ }^{4}$ Geneviève Delaisi de Parseval, Suzanne Lallemand, L'art d'accommoder les bébés, 100 ans de recettes françaises de puériculture (Paris: Seuil, 1980).

${ }^{5} \mathrm{Si}$ l'on excepte la magnifique salle de bains que Samuel Marchak et Vladimir Lebedev imaginent dans Hier et Aujourd'hui en 1925 pour célébrer les progrès de la société nouvelle. (Saint-Pétersbourg: Raduga, 1925; récemment édité en français dans une traduction de Françoise Morvan et André Markowicz: Nantes: MeMo, 2005).

${ }^{6}$ Wilhelm Bush, Max und Moritz, eine Bubengeschichte in sieben Streichen (München: Braun und Schneider, 1865).

${ }^{7}$ Heinrich Hoffmann, Lusstige Geschichten und drollige Bilder mit 15 schön kolorierten Tafeln für Kinder von 3 bis 6 Jahren (Frankfurt, 1845).

${ }^{8}$ Elsa Beskow, Petter och Lotta på äventyr, (BonnierCarlsen, 1929).

${ }^{9}$ Elle rejoint ainsi le lit, la cuisine, la cachette sous la table, plus tard aussi le fauteuil.

10 "Dans les baignoires isolées chaque élève ne peut avoir un surveillant [é ]. Il songe au mal dans l'isolement. Il y est excité par l'influence de l'eau chaude. Les bains chauds ne sont bons au collège que pour les malades que l'on ne quitte pas un seul instant." C.Pavet de Courteille, Hygiène des collèges et des maisons d'éduducation, Paris: 1827. Cité par Georges Vigarello, op.cit., p.189.

${ }^{11}$ le livre mesure 27x 8,5 cm; dans l'édition originale, il est vendu dans un coffret imitant le bois.
} 
${ }^{12}$ Tomi Ungerer, Emile (1960 ; traduction française Paris :Planète, 1968).

${ }^{13}$ Sarah la petite tsigane (autre titre: Sarah petite fille du voyage) raconté par Gaby Cozain, illustré par May Angeli (Paris: Flammarion, Les albums du Père Castor, 1972).

${ }^{14}$ Pericle Luigi Giovannetti, Max, 1954 (Paris: L'Ecole des Loisirs, 2003).

${ }^{15}$ Kay Thompson, Eloïse, ill. Hilary Knight, traduit de l'américain par Jean-François Ménard, p.99 (Paris: Gallimard, 1982; édition originale 1955).

${ }^{16}$ Arnold Lobel, Porculus, 1968 (Paris: L'école des loisirs, traduit de l'américain par Adolphe Chagot, 1971).

${ }^{17}$ Eugène Ionesco, Conte $n^{\circ} 3$ pour enfants de moins de trois ans, ill. Philippe Corentin (Paris, Un livre Harlin Quist, 1971). Notons que dans le Conte $n^{\circ} 4$ Ionesco met en scène le père de Josette cherchant à égarer la fillette le temps de faire sa toilette en toute tranquillité; l'album, illustré par Nicole Claveloux, est parsemé d'images du père entièrement nu.

${ }^{18}$ Max Howard, Toute ressemblance avec des personnages existant ou ayant existé n'est pas une pure coüncidence, ill. Monique Gaudriault et Jacques Rozier pour cette image (Paris: Encore un livre d'Harlin Quist, 1974).

${ }^{19}$ Marie-France Boyer, La grippe de Nils, ou la famille éclatée, ill. Marie Gard (Paris: Des Femmes, 1980).

${ }^{20}$ Felicia Bond, Poinsettia ne veut plus de sa famille Harper and Row, 1981 (Paris, Joie de Lire / Ecole des loisirs, 1985).

${ }^{21}$ Wendy Smith, Théodore souris, fils unique (Paris: Flammarion, 1986).

${ }^{22}$ Françoise Kerisel, Moi, Matthieu, j'habite chez mon père, Ill Alain Gauthier (Paris: Ipomée-Albin Michel, 1991).

${ }^{23}$ Jill Murphy, Le Bain de madame Trompette (Paris: Bayard éditions, 1986; édition originale: Five minutes' peace, London, 1986).

${ }^{24}$ J. Van Leeuwen, Nouvelles histoire d'Olivier Cochon, ill. Arnold Lobel (Paris: Joie de lire / école des loisirs, 1986; édition originale 1981).

${ }^{25}$ Steven Kellogg, Le Têtard mystérieux (Paris: Lotus, 1979. Editons originale 1977).

${ }^{26}$ Martha Alexander, Pas de canard dans ma baignoire, (Duculot, 1984 ; édition originale1973).

${ }^{27}$ Grégoire Solotareff, Kiki la souris (Paris: Ecole des Loisirs, 1988).

${ }^{28}$ Claude Ponti, Pétronille et ses 120 petits (Paris: Ecole des loisirs, 1990).

${ }^{29}$ Antoon Krings, Zaza au bain (Paris: Ecole des loisirs, 1992).

${ }^{30}$ Martine Toulza, Un loup dans la baignoire, ill. Claire Le Grand (Toulouse: Milan, 2001).

${ }^{31}$ Rosemary Wells, Le bain de Max (Paris: Ecole des loisirs, 1985; ).

${ }^{32}$ Elzbieta, Cornefolle (Paris: Pastel, 1989).

${ }^{33}$ Elzbieta, L'Enfance de l'art (Rodez: Editions du Rouergue, 1997) p.185.

${ }^{34}$ John Burningham, Veux-tu sortir du bain, Marcelle? (Paris: Flammarion, Père Castor, 1978; édition originale Time to get out of the bath, Shirley, London: 1978).

${ }^{35}$ Anaïs Vaugelade, Zuza dans la baignoire (Paris: Ecole des loisirs, 1998).

${ }^{36}$ Claude Ponti, Une semaine de Monsieur Monsieur (Paris: Ecole des loisirs, 1999).

${ }^{37}$ Anne Brouillard, Promenade au bord de l'eau (Paris: Editions du Sorbier, 1996).

${ }^{38}$ Philippe Dumas, Laura perd la tête (Paris: Ecole des loisirs, 1981).

${ }^{39}$ Isabelle Nières, ñFictions pour enfants, petites mythologies des originesò Horizonte und Grenzen,

Kolloquium 25 Jahre Schweizerisches Jugendbuch Institut (Zürich: 1994). 